\title{
The Influence of Salt Sensitivity Phenotype on Sodium Excretion and Diuresis: A Chrononutrition Pilot Study
}

\author{
Upeksha Sewwandi Alwis ${ }^{(D},{ }^{1}$ Irina Verbakel, ${ }^{1}$ Kim Pauwaert, ${ }^{1}$ Joris Delanghe, ${ }^{2}$ \\ Lien Dossche, ${ }^{3,4}$ John Van Camp, ${ }^{5}$ Saskia Roggeman, ${ }^{6}$ and Karel Everaert ${ }^{1}$ \\ ${ }^{1}$ Department of Human Structure and Repair, Ghent University, 9000 Ghent, Belgium \\ ${ }^{2}$ Department of Diagnostic Sciences, Ghent University, 9000 Ghent, Belgium \\ ${ }^{3}$ Department of Internal Medicine and Pediatrics, Ghent University, 9000 Ghent, Belgium \\ ${ }^{4}$ Department of Pediatric Nephrology, Ghent University, 9000 Ghent, Belgium \\ ${ }^{5}$ Department of Food Technology, Safety and Health, Ghent University, 9000 Ghent, Belgium \\ ${ }^{6}$ Research and Policy Department, Psychiatric Center Sint-Jan-Baptist, 9060 Zelzate, Belgium
}

Correspondence should be addressed to Upeksha Sewwandi Alwis; upekshasewwandi.alwis@ugent.be

Received 29 September 2021; Accepted 6 December 2021; Published 31 January 2022

Academic Editor: Ahmed Abdelwahab

Copyright (C) 2022 Upeksha Sewwandi Alwis et al. This is an open access article distributed under the Creative Commons Attribution License, which permits unrestricted use, distribution, and reproduction in any medium, provided the original work is properly cited.

\begin{abstract}
Background. Chrononutrition studies on interaction of diet/nutrients on endogenous circadian clocks and meal timing on metabolic homeostasis may be of importance in the management of nocturnal polyuria (NP), owing to loss of circadian rhythm in nighttime urination. Dietary salt restriction is an increasingly popular lifestyle recommendation for NP patients. Aim. This study aims to evaluate the effect of an acute salt load on diuresis and to study the phenomenon of salt sensitivity. Methodology. Young, healthy men $(n=21$, fasted and sober) ingested $500 \mathrm{ml}$ of water on the control day and $8 \mathrm{~g}$ and $12 \mathrm{~g}$ of salt with water (500 ml) on two other days. Blood and urine samples were collected at $0 \mathrm{hrs}, 2 \mathrm{hrs}$, and $4 \mathrm{hrs}$ and voided volumes were recorded. Diuresis, serum and urine osmolality, sodium, potassium, urea, and creatinine were measured. Salt sensitivity was determined based on the rate of sodium excretion. Results. Compared to $8 \mathrm{~g}$, ingestion of $12 \mathrm{~g}$ of salt significantly increased diuresis after 4 hrs. Pure water load induced fast diuresis, whereas salt and water load initially reduced diuresis and promoted late increase in diuresis. The total voided volume was significantly lower in the salt sensitive individuals. Conclusion. Taken together, salt sensitivity profile and type and time of fluid intake are important considerations to build effective personalized lifestyle recommendations for NP patients, which needs further investigation.
\end{abstract}

\section{Introduction}

A wide range of biological and physiological functions in the body follow circadian rhythms, which are orchestrated by the circadian clocks present in many organs and cells, including brain, kidney, and bladder [1]. Over the last few years, a growing body of evidence has emerged on the interaction of diet/nutrients and meal timing on metabolism and endogenous circadian rhythm [2-4]. This area of "Chrononutrition" may also be of importance in the management of nocturia, in relation to loss of circadian rhythm in urine production [5]. Nocturia or the act of waking up at night to urinate during the main sleeping period [6] is a very common and bothersome lower urinary tract symptom (LUTS), and it is most common in the elderly [7]. Nighttime voiding in healthy humans is regulated through circadian rhythms, by decreasing brain arousal and kidney urine production rate, whilst increasing functional bladder capacity, which all three combined, prevents nocturnal micturition. In most patients suffering from nocturia, disturbances in this circadian rhythm are shown [1].

In an in vivo study, Gizowski et al. showed that an acute salt load could alter the circadian clock timing and could drive unscheduled homeostatic responses via clock-output networks [8]. On the other hand, excessive dietary salt intake has been identified as a risk factor for nocturia [9] and dietary salt 
reduction significantly improved nocturia in patients with high salt intake. Thus, the effect of high salt intake in nocturia patients has garnered a great attention in the management of nocturia [10-12]. However, individuals respond differently to an excess dietary salt intake, and this variability has been extensively studied in the phenomenon of salt sensitivity of blood pressure (BP) [13]. Salt sensitive BP has been explained by various mechanisms including sodium retention, blood volume expansion, abnormalities in renin-angiotensin-aldosterone-system (RAAS) and altered renal function with impact on sodium and fluid homeostasis [14-16]. Thus, salt sensitivity contains also the inability of the kidney to excrete excess sodium [17]. Yet, to our understanding, salt sensitivity has not been studied in the context of the renal ability to excrete sodium and water followed by a high salt load.

In our previously published study, haptoglobin (Hp) polymorphism-related salt sensitivity in sodium and water handling were reported [18]. In this study, we hypothesized that healthy individuals who display a low rate of sodium excretion after an acute high salt load react inappropriately with salt and water retention and are therefore called "saltsensitive". Understanding these individual differences based on the ability to excrete excess sodium after a high salt load and its effect on diuresis and other main urinary solutes such as urea could lead to individualized lifestyle interventions and recommendations for nocturia patients. Therefore, the aim of this study was to evaluate the effect of an acute salt load on diuresis, sodium, and urea excretion, in order to study the phenomenon of salt sensitivity.

\section{Materials and Methods}

2.1. Study Population. Twenty-one young, healthy male volunteers with median age of 26 years (21-27) and BMI $23 \mathrm{~kg} / \mathrm{m}^{2}(20-24)$ participated in the study. The subjects were recruited through the university and the community via posters and flyers.

2.1.1. Baseline Standardization and Exclusion Criteria. Young, healthy male volunteers were recruited to avoid any kind of interference regarding sodium and fluid homeostasis. Therefore, participants taking any type of medication or suffering from a chronic condition were excluded. All the trials were conducted after an overnight fasting period and a sober state in the morning to avoid the influence of diet and differences on hydration status. The subjects were not allowed to consume any alcoholic beverages for $24 \mathrm{~h}$ and were on their normal diet prior to each test day. All the subjects recorded a 3 day food diary including 2 week days and 1 weekend day, which used to evaluate their normal dietary intake.

2.2. Study Design. This is a prospective, interventional, pilot study conducted between June 2020 and December 2020. The study protocol was approved by the Ghent University Hospital Ethics Committee (EC 2019/0710; Clinical trial registration No: NCT04526340) and conducted in accordance with the Declaration of Helsinki. Written informed consent was obtained from all subjects.
2.2.1. Experimental Protocol. In this study setting, each individual, acting as its own control, came to the hospital for three different tests on three different mornings. On the control day, the participants ingested $500 \mathrm{~mL}$ of still water over 15 minutes. On the 2 salt test days, the volunteers ingested either 8 or 12 salt capsules ( $1 \mathrm{~g} /$ capsule), along with $500 \mathrm{~mL}$ still water in 15 minutes (Figure 1).

In between testing days, there were at least two days to reduce interference from the previous high dose salt intake. The mineral composition of still water used (EVIAN RPET water bottle $500 \mathrm{~mL}$ ) is presented in Table 1.

2.2.2. Urine and Blood Collection. Upon arrival at the hospital, the volunteers delivered their first morning urine in a measuring cup and a urine sample was taken (baseline urine sample: U1). Afterwards, a $15 \mathrm{~mL}$ serum blood sample (B1) was collected. The height and the weight of the subjects were taken after the urine and blood samples were collected. Participants were asked to void again after 2 hours. Urine volume was registered and a second urine (U2) and a blood sample (B2) were taken. At the end of $4^{\text {th }}$ hour of the study period, the final urine sample (U3) was collected, after registration of the voiding volume.

2.3. Urine and Serum Analysis. Voided volumes, urine osmolality, and sodium, potassium, urea, and creatinine levels were analyzed from each urine sample. Osmolality was measured using freezing-point depression (OSMO station OM-6060, Arkray) [19]. Creatinine was measured using a compensated rate-blanked alkaline picrate method [20]. Urea was measured using an enzymatic assay (urease/glutamate dehydrogenase method) [21]. Sodium and potassium was measured using indirect potentiometry (Cobas 8000 modular analyzer, Roche) [22].

2.3.1. Calculation of Renal Clearances. Serum osmolality was determined and utilized to calculate solute clearance (urine osmolality $\times$ urine flow/plasma osmolality $_{\text {) and free water }}$ clearance (FWC) (urine flow-solute clearance).

2.3.2. Calculation of Salt Sensitivity. Salt sensitivity was defined as alterations in sodium excretions due to high salt load. Therefore, to calculate the salt sensitivity of sodium excretion of each subjects after the high salt load, following equation was used:

$$
\begin{aligned}
\text { salt sensitivity } & =\text { derivative }\left[\frac{\Delta \text { sodium excretion }}{\Delta \text { sodium intake }}\right], \\
(\text { a }) & =\text { slope of the linear function }\left[\frac{\Delta y}{\Delta x}\right] .
\end{aligned}
$$

2.4. Salt Sensitivity Phenotyping. A secondary analysis to calculated salt sensitivity was carried out by dividing the study population into three groups/phenotypes on the basis 


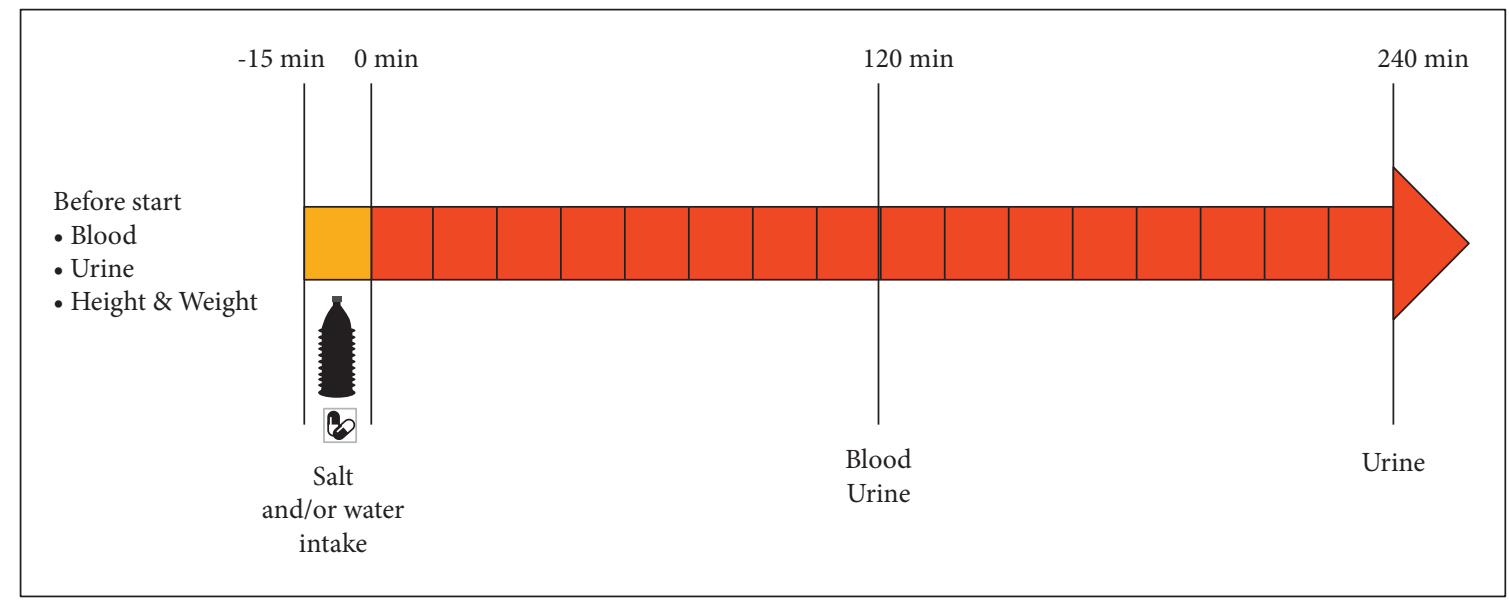

Figure 1: Diagram of experimental protocol.

TABLE 1: Mineral composition of water obtained from the water bottle label.

\begin{tabular}{cccccccccc}
\hline & Sodium & Potassium & Calcium & Magnesium & Nitrates & Bicarbonates & Sulfates & Silica & Chlorides \\
\hline Unit $\mathrm{mg} / \mathrm{L}$ & 6.5 & 1 & 80 & 26 & 3.8 & 360 & 14 & 15 & 10 \\
\hline
\end{tabular}

of the derived value of rate of sodium excretion; $<0$ : salt sensitive (SS), 0 to 0.99: moderate sensitive ( $S M)$, or $\geq 1$ : salt resistant (SR).

\subsubsection{Haptoglobin (Hp) Phenotyping and Plasma Hp} Concentration. Starch gel electrophoresis was carried out for Haptoglobin phenotyping of haemoglobin-supplemented serum as described by Smithies et al. [23]. Hydrolysed starch (11.5\%) was used to prepare the starch gel in a $0.1 \mathrm{~mol} / 1$ Tris-citrate buffer $(\mathrm{pH} 8.86)$. Electrophoresis was performed for $1 \mathrm{~h}$ at $200 \mathrm{~V}$ in a $0.3 \mathrm{~mol} / \mathrm{l}$ borate buffer ( $\mathrm{pH}$ 8.4). Hp-Hb complexes were visualized using metalenhanced peroxidase reagents.

Plasma Hp concentration was measured by fixed-time immuno-nephelometry using Dade Behring rabbit antihuman $\mathrm{Hp}$ polyclonal antibodies [24]. The Hp assay was standardized using CRM 470 reference material [25].

2.5. Calculation of Dietary Sodium Intake. Three day food diaries were used to calculate dietary sodium intake of each individuals using the sodium content of each food based on Belgian food composition database (Internubel.be).

2.6. Statistical Analysis. Clinical and biochemical parameters between the three test days were compared using the Wilcoxon signed-rank test for two related samples. Within a test day, variance over $4 \mathrm{hrs}$ was compared using the related-samples Friedman's Two-Way Analysis of variance, followed by a pairwise comparison with adjusted $p$-values by the Bonferroni correction for multiple tests. Differences in the salt sensitivity phenotype were compared using Kruskal-Wallis Test for $\mathrm{K}$ independent samples. Multiple regression analysis was used to determine the relationship between the total voided volume ( $\mathrm{ml} / 4 \mathrm{hrs})$, age (years), BMI $\left(\mathrm{kg} / \mathrm{m}^{2}\right)$, dietary sodium intake $(\mathrm{g} /$ day), salt sensitivity phenotype, Hp phenotype (Hp 1-1 vs. rest), Hp concentration $(\mathrm{mmol} / \mathrm{L})$, and urinary excretion of potassium, urea, and creatinine ( $\mathrm{mmol} / 4 \mathrm{hrs})$.

All continuous variables are reported as median (interquartile range). All analyses were performed using IBM SPSS Statistics for Windows, version 26 (IBM Corp., Armonk, NY., USA). A $p$-value $<0.05$ was deemed statistically significant.

\section{Results}

3.1. Study Population. In the total 21 subjects participated in the study, 19 subjects completed all 3 test days. Two subjects voluntary withdrew after completing the control and $8 \mathrm{~g}$ salt day, due to discomfort caused by salt ingestion. Baseline characteristics are presented in Table $2.42 .9 \%$ of the population had $\leq 3.2 \mathrm{~g}$ dietary sodium intake (= moderate, $\leq 8 \mathrm{~g}$ salt/per day [26]) per day. Only $14 \%$ of the study population met the WHO recommendation of $<2 \mathrm{~g}$ dietary sodium/day. Hp phenotypic distribution of the study population comprised $38 \%$ Hp 1-1, 24\% Hp 2-2 and 38\% Hp 2-1. Body weight, serum osmolality, urine osmolality, or diuresis rate did not significantly differ between the trial days for each participant.

\subsection{Effect of Salt and/or Water Ingestion on Fasted, Sober Healthy Young Men}

3.2.1. Before and after Effect. Control day: After the water load, increased diuresis rate and reduced urine osmolality were observed compared to baseline, and the effect was more prominent at $2 \mathrm{hrs}$ (Table 3: A). However, no significant difference was observed in FWC or in urinary sodium/ creatinine ratio at any time. On the other hand, compared to baseline, urinary potassium/creatinine ratio was significantly greater at all-time points, whereas urinary urea/creatinine ratio significantly increased at $2 \mathrm{hrs}$. 
TABLE 2: Baseline demographics of the population.

\begin{tabular}{|c|c|c|c|c|}
\hline Parameter & \multicolumn{4}{|c|}{ Results $(n=21)$} \\
\hline Age (years) & \multicolumn{4}{|c|}{$26(21-27)$} \\
\hline BMI $\left(\mathrm{kg} / \mathrm{m}^{2}\right)$ & \multicolumn{4}{|c|}{$23.6(20.5-24.7)$} \\
\hline Dietary sodium intake (g/day) & \multicolumn{4}{|c|}{$3.89(2.51-4.79)$} \\
\hline Moderate intake $\leq 3.2 \mathrm{~g} /$ day $(\%)$ & \multicolumn{4}{|c|}{$9(42.9)$} \\
\hline High intake $>3.2 \mathrm{~g} /$ day $(\%)$ & \multicolumn{4}{|c|}{$12(57.1)$} \\
\hline \multicolumn{5}{|l|}{ Haptoglobin phenotype (\%) } \\
\hline $1 \mathrm{Hp} 1-1$ & \multicolumn{4}{|c|}{$8(38)$} \\
\hline $2 \mathrm{Hp} \mathrm{2-1}$ & \multicolumn{4}{|c|}{$8(38)$} \\
\hline 3 Hр 2-2 & \multicolumn{4}{|c|}{$5(24)$} \\
\hline \multicolumn{5}{|l|}{ Salt sensitivity phenotype (\%) } \\
\hline $1 \mathrm{SS}$ & \multicolumn{4}{|c|}{$6(31.6)$} \\
\hline $2 \mathrm{SM}$ & \multicolumn{4}{|c|}{$12(63.1)$} \\
\hline \multirow{2}{*}{$3 \mathrm{SR}$} & \multicolumn{4}{|c|}{$1(5.3)$} \\
\hline & Day 1 (n-21) & Day $2(n=21)$ & Day $3(n=19)$ & $P$ value \\
\hline Body weight (kg) & $75(68.3-80.5)$ & $74(68.7-80.5)$ & $73.3(68-79)$ & NS \\
\hline \multicolumn{5}{|l|}{ Plasma } \\
\hline Osmolality $(\mathrm{mOsm} / \mathrm{Kg})$ & $287(285-290)$ & $288(288-291)$ & $288(286-291)$ & NS \\
\hline Sodium/Creatinine $(\mathrm{mmol} / \mathrm{mmol})$ & $153(138-170)$ & $152(146-160)$ & $150(141-159)$ & NS \\
\hline Potassium/Creatinine $(\mathrm{mmol} / \mathrm{mmol})$ & $4.5(4-5)$ & $4.3(4.1-4.6)$ & $4.4(4-4.5)$ & NS \\
\hline Urea/Creatinine $(\mathrm{mmol} / \mathrm{mmol})$ & $35(29-44)$ & $35(33-43)$ & $35(29-42)$ & NS \\
\hline \multicolumn{5}{|l|}{ Urine } \\
\hline Diuresis rate $(\mathrm{mL} / \mathrm{min})$ & $0.8(0.4-0.9)$ & $0.5(0.3-0.8)$ & $0.4(0.3-0.6)$ & NS \\
\hline Osmolality $\mathrm{mOsm} / \mathrm{kg}$ & $842(713-1001)$ & $951(840-1039)$ & $988(596-1049)$ & NS \\
\hline Sodium/Creatinine $(\mathrm{mmol} / \mathrm{mmol})$ & $6.8(5.4-13.4)$ & $5.2(3.6-7.8)$ & $6.2(5.7-7.3)$ & NS \\
\hline Potassium/Creatinine $(\mathrm{mmol} / \mathrm{mmol})$ & $4(2-5.6)$ & $3.9(2.5-4.8)$ & $3(1.9-7)$ & NS \\
\hline Urea/Creatinine $(\mathrm{mmol} / \mathrm{mmol})$ & $27(22-31)$ & $26(20-29)$ & $24(20-32)$ & NS \\
\hline
\end{tabular}

$* p<0.05$ for control vs. $8 \mathrm{~g}$ salt, $\dagger p<0.05$ for control vs. $12 \mathrm{~g}$ salt and $\Delta p<0.05$ for $8 \mathrm{~g}$ vs. $12 \mathrm{~g}$ salt; Wilcoxon signed rank test. BMI $=$ body mass index; $\mathrm{Hp}=$ haptoglobin phenotype; $\mathrm{SS}=$ salt sensitive; $\mathrm{SM}=$ moderate sensitive; $\mathrm{SR}=$ salt resistant; $\mathrm{NS}=$ not significant. This study was conducted in 3 different test days as day 1,2 , and 3 . Therefore, clinical parameters of plasma and urine samples taken at the baseline/before the test started on different test days (as day 1,2 , and 3) are shown in the bold values.

TABle 3: Time course of changes in diuresis rate, urine osmolality, FWC, and solute/creatinine ratios within the test days (A) and between the test days (B), over $4 \mathrm{hrs}$ after salt and/or water ingestion.

\begin{tabular}{|c|c|c|c|}
\hline \multicolumn{4}{|c|}{ (A) Within the test days } \\
\hline \multicolumn{4}{|c|}{ Diuresis $(\mathrm{mL} / \mathrm{min})$} \\
\hline 2 hrs & $3.5(1.4-4.3)$ & $1.1(0.8-1.5)$ & $1.2(0.8-1.8)$ \\
\hline $4 \mathrm{hrs}$ & $1.3(0.8-2.0)$ & $1.2(0.8-1.5)$ & $1.6(1.0-1.9)$ \\
\hline \multirow{2}{*}{$P$ value } & Baseline vs. 2 hrs: $<0.001 \mathrm{a}$ & Baseline vs. 2 hrs: $0.006 \mathrm{a}$ & Baseline vs. 2 hrs: $0.006 a$ \\
\hline & Baseline vs. 4 hrs: $0.026 a$ & Baseline vs. 4 hrs: $0.021 \mathrm{a}$ & Baseline vs. 4 hrs: $0.001 \mathrm{a}$ \\
\hline \multicolumn{4}{|c|}{ Urine osmolality $(\mathrm{mOsm} / \mathrm{kg})$} \\
\hline $2 \mathrm{hrs}$ & $260(166-910)$ & $927(824-1072)$ & $905(771-1056)$ \\
\hline $4 \mathrm{hrs}$ & $517(384-742)$ & $900(796-982)$ & $849(764-960)$ \\
\hline$P$ value & $<0.001^{* *}$ & NS & NS \\
\hline \multicolumn{4}{|c|}{ Free water clearance $(\mathrm{mL} / \mathrm{min})$} \\
\hline $2 \mathrm{hrs}$ & $0.3(-2.6-1.4)$ & $-2.2(-3.0-(-1.5))$ & $-2.5(-3.3-(-1.8))$ \\
\hline $4 \mathrm{hrs}$ & $-1.2(-1.7-(-0.6))$ & $-2.7(-2.9-(-1.5))$ & $-2.8(-3.5-(-1.9))$ \\
\hline & & Baseline vs. 2 hrs: $0.006 a$ & Baseline vs. 2 hrs: $<0.001 \mathrm{a}$ \\
\hline$P$ value & NS & Baseline vs. 4 hrs: $0.021 \mathrm{a}$ & Baseline vs. 4 hrs: $0.002 \mathrm{a}$ \\
\hline \multicolumn{4}{|c|}{ Sodium/creatinine $(\mathrm{mmol} / \mathrm{mmol})$} \\
\hline $2 \mathrm{hrs}$ & $7.8(6.3-12.7)$ & $12.4(5.8-16.9)$ & $13.1(11.6-20.5)$ \\
\hline $4 \mathrm{hrs}$ & $10.2(6.5-16)$ & $23.6(16.2-31.4)$ & $30.6(26.3-38.6)$ \\
\hline$P$ value & NS & $<0.001^{* *}$ & $<0.001^{* *}$ \\
\hline \multicolumn{4}{|c|}{ Potassium/creatinine $(\mathrm{mmol} / \mathrm{mmol})$} \\
\hline $2 \mathrm{hrs}$ & $5.7(3.9-8.5)$ & $5.8(4.1-7.8)$ & $6.2(3.5-7.7)$ \\
\hline $4 \mathrm{hrs}$ & $9.4(6.2-11)$ & $13(9.7-15.1)$ & $12.5(9.3-17.7)$ \\
\hline & Baseline vs. 2 hrs: $0.001 \mathrm{a}$ & Baseline vs. 4 hrs: $<0.001 \mathrm{a}$ & Baseline vs. 4 hrs: $<0.001 \mathrm{a}$ \\
\hline$P$ value & Baseline vs. 4 hrs: $<0.001 \mathrm{a}$ & 2 hrs vs. 4 hrs: $0.003 a$ & 2 hrs vs. 4 hrs: $0.004 \mathrm{a}$ \\
\hline \multicolumn{4}{|c|}{ Urea/creatinine $(\mathrm{mmol} / \mathrm{mmol})$} \\
\hline $2 \mathrm{hrs}$ & $30(25-32)$ & $25(21-32)$ & $28(20-34)$ \\
\hline $4 \mathrm{hrs}$ & $28(23-33)$ & $29(22-36)$ & $28(24-35)$ \\
\hline$P$ value & Baseline vs. 2 hrs: $0.005 \mathrm{a}$ & Baseline vs. 4 hrs: $0.018 \mathrm{a}$ & Baseline vs. 4 hrs: $0.024 \mathrm{a}$ \\
\hline
\end{tabular}


TABLE 3: Continued.

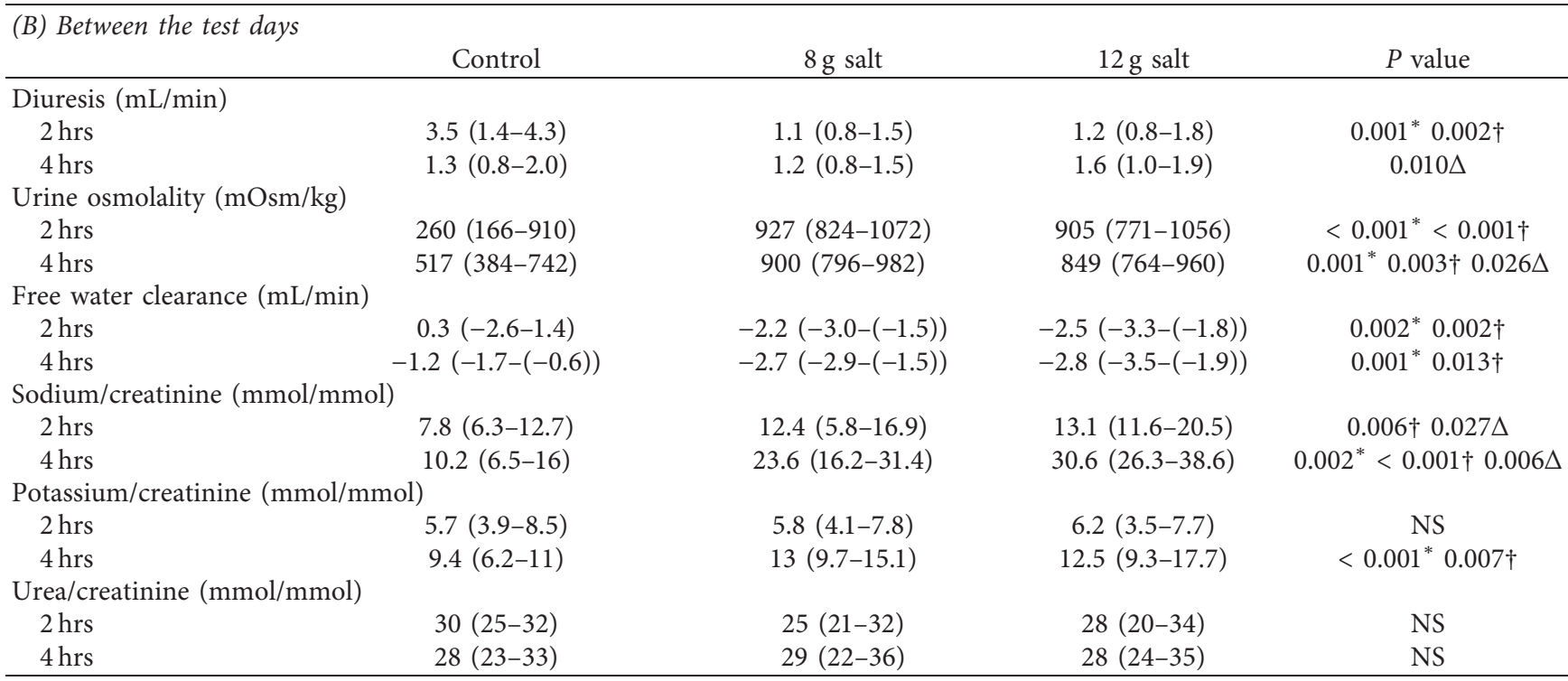

${ }^{* *} p<0.05$ for related-samples Friedman's Two-Way Analysis of variance and a $p<0.005$ for pairwise comparison with adjusted $p$ values by the Bonferroni correction for multiple tests. NS $=$ not significant. $* p<0.05$ for control vs. $8 \mathrm{~g}$ salt, $\uparrow p<0.05$ for control vs. $12 \mathrm{~g}$ salt and $\Delta p<0.05$ for $8 \mathrm{~g}$ vs. $12 \mathrm{~g}$ salt; Wilcoxon signed rank test. NS = not significant.

Salt days: After the salt load, compared to baseline, diuresis rate significantly increased for both salt dosages, but more profoundly for $12 \mathrm{~g}$ salt day at $4 \mathrm{hrs}$. However, urine osmolality showed no significant difference, whereas FWC reduced dramatically. Urinary sodium increased significantly, again more profoundly for $12 \mathrm{~g}$ of salt. A significant increase in urinary potassium and urea was observed only 4 hrs after ingestion of salt (Table 3: A).

3.2.2. Salt Effect. Control vs salt days: Two hours after ingesting the salt, diuresis rate reduced significantly while increasing urinary osmolality. FWC reduced significantly and urinary sodium levels were significantly higher in the $12 \mathrm{~g}$ salt group, but not in the $8 \mathrm{~g}$. In addition, ingestion of salt did not affect the urinary potassium or urea measured at 2 hrs (Table 3, B).

At $4 \mathrm{hrs}$, diuresis rate did not significantly differ between salt days and the control day. Nevertheless, increased urinary osmolality was observed in both salt groups, together with a significantly lower FWC. Urinary sodium and potassium were significantly higher in both salt trials than in the control, and similar to $2 \mathrm{hrs}$, ingestion of salt did not affect urinary urea at $4 \mathrm{hrs}$ (Table $3, \mathrm{~B}$ ).

3.2.3. Dose Effect. $8 \mathrm{~g}$ salt vs. $12 \mathrm{~g}$ salt: Compared with the $8 \mathrm{~g}$ of salt, $12 \mathrm{~g}$ of salt significantly increased diuresis and reduced urinary osmolality 4 hours after ingestion. $12 \mathrm{~g}$ of salt significantly increased urinary sodium at all times, but more intensely at $4 \mathrm{hrs}$ (Table 3, B). No significant changes were observed in FWC or urinary potassium and urea levels between the two salt dosages. Figure 2 shows the trends of fluid balance over the time on 3 different test days.
3.3. Relationship between Total Voided Volumes and Salt Sensitivity Phenotype. Comparison between the 3 salt sensitivity phenotypes showed that subjects with a higher rate of sodium excretion, further stated as salt sensitivity phenotype SM and SR, had significantly greater total voided volumes than SS subjects (SR: 480 and SM: 405 (320-450) vs. SS: 265 (197-300) $\mathrm{ml} / 4 \mathrm{hrs}, p=0.021 *)$. In multiple regression analysis, total voided volume produced after a high salt load $(12 \mathrm{~g})$, was positively associated with salt sensitivity phenotype, age, $\mathrm{Hp}$ phenotype, and creatinine excretion, whereas urea excretion was associated inversely with the total voided volume (Table 4).

\section{Discussion}

4.1. Summary of Evidence. The present study aimed to investigate the effect of an acute salt load on urine production and body fluid balance. One of the main findings of our study is that, in healthy young men, an acute high salt load (12 g) increased diuresis significantly compared to a moderate salt load $(8 \mathrm{~g})$, but the effect on diuresis was delayed by $4 \mathrm{hrs}$ after ingestion. The total voided volume was significantly lower in the salt-sensitive phenotype, reflecting the subjects with a low rate of sodium excretion. Furthermore, a larger voided volume was associated with an increased rate of urinary sodium and creatinine excretion, age, Hp2-2/2-1 phenotypes, and decreased urea excretion. Therefore, our present study results suggest that an individual's ability to excrete excess urinary sodium is a main determinant of urine output following a high salt load.

The renal concentrating ability to excrete dietary sodium following a dietary salt load has been investigated earlier under different hydration and salt intake levels [27-29]. A study done by Rakova et al. reported an increased urinary 


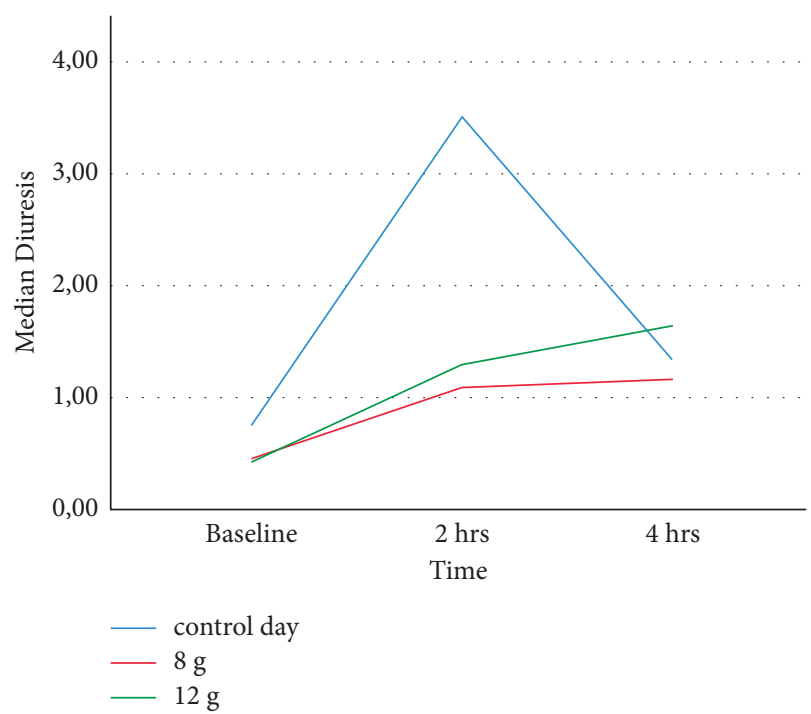

(a)

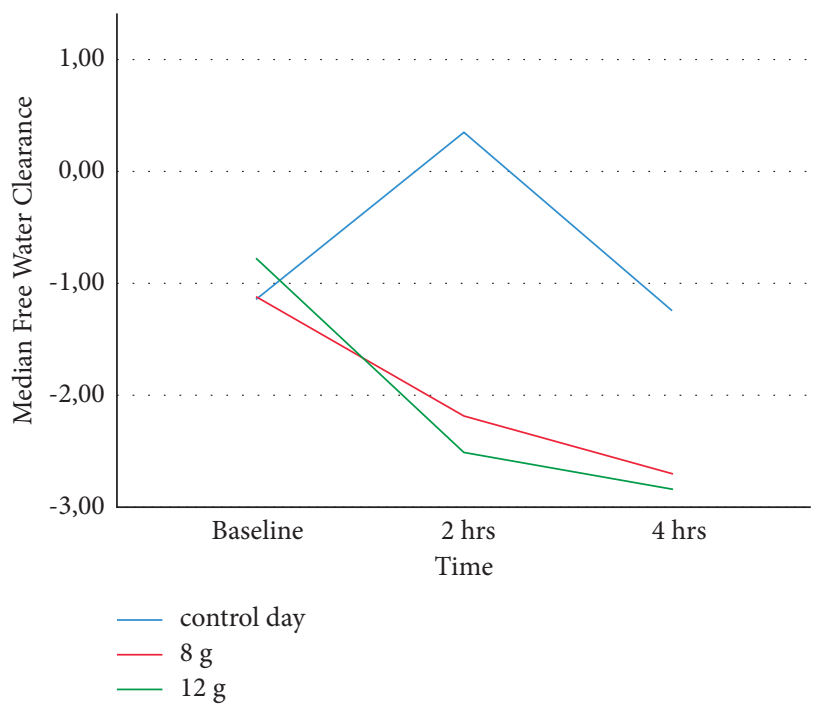

(c)

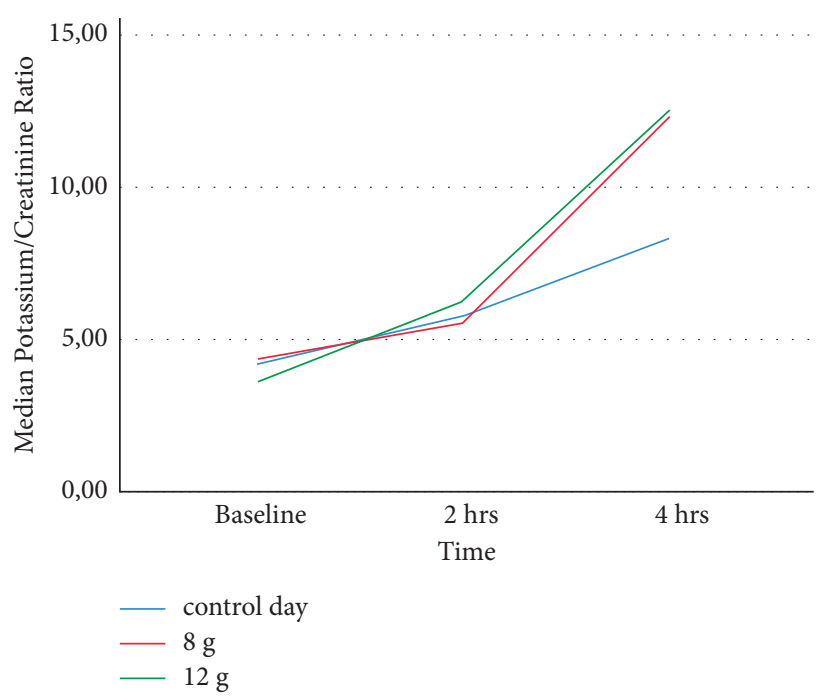

(e)

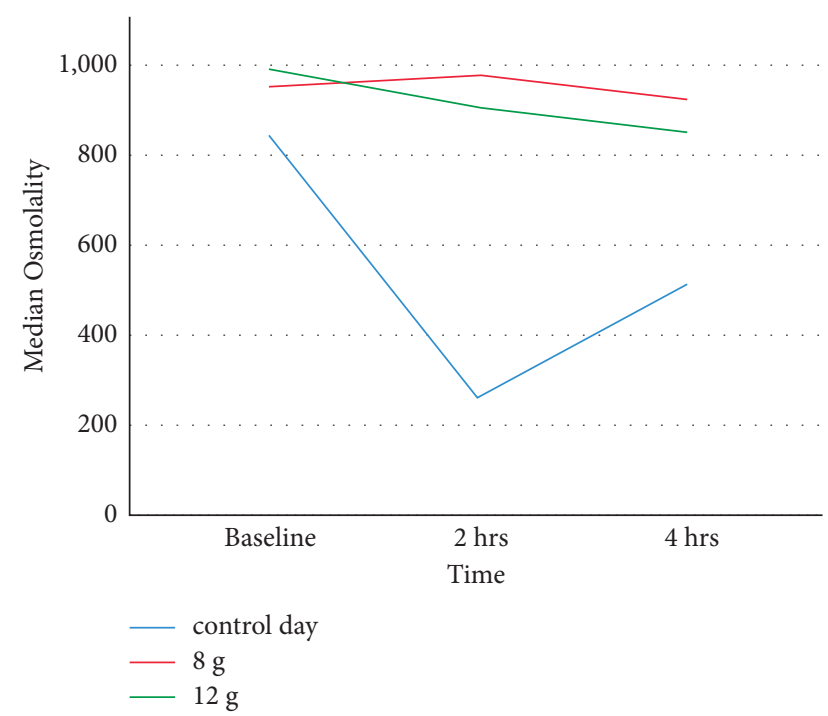

(b)

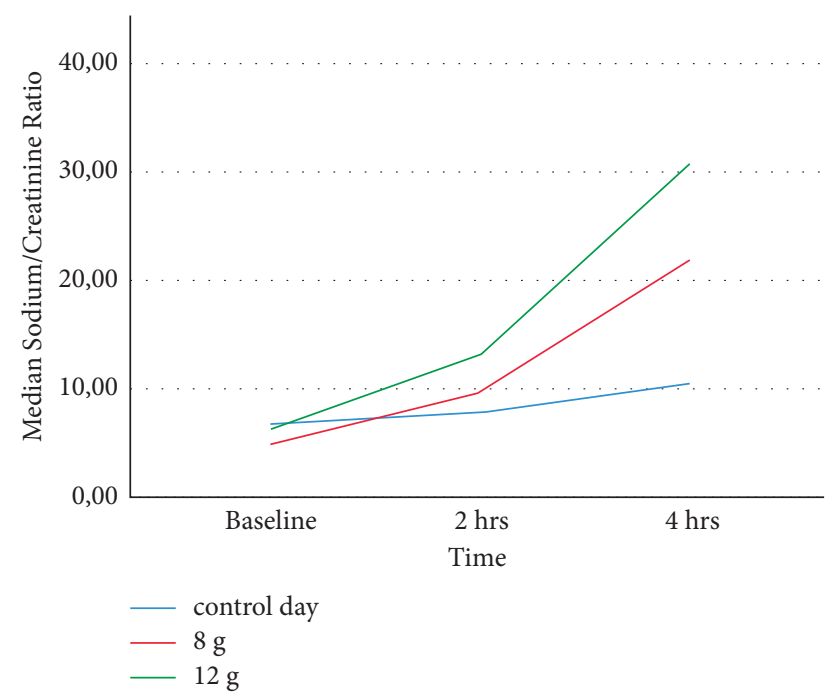

(d)

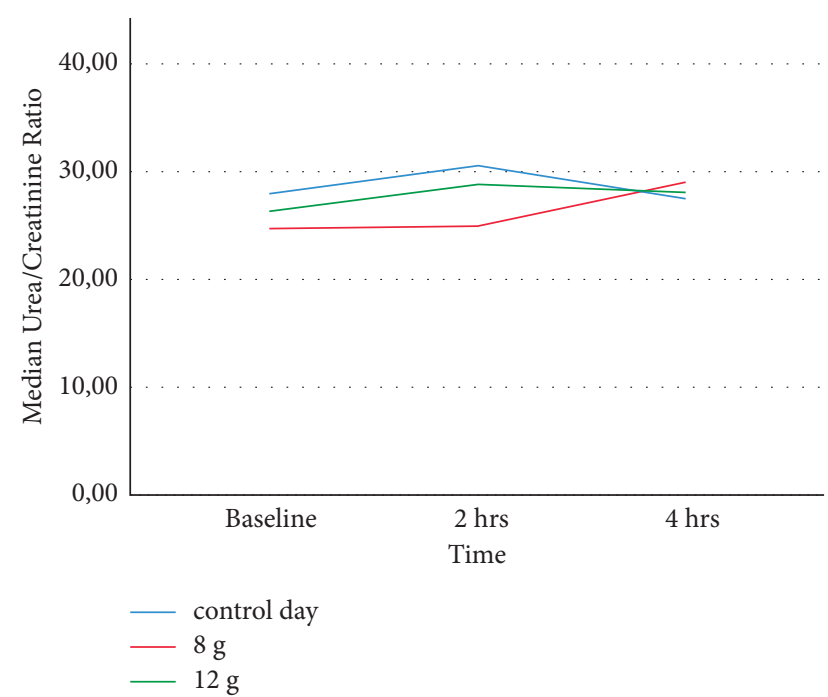

(f)

Figure 2: Trend of fluid balance for the 3 different test days. Diuresis rate $\mathrm{ml} / \mathrm{min}$ (a), urine osmolality $\mathrm{mOsm} / \mathrm{kg}(\mathrm{b})$, free water clearance $\mathrm{ml} / \mathrm{min}(\mathrm{c})$, sodium/creatinine $(\mathrm{mmol} / \mathrm{mmol})(\mathrm{d})$, potassium/creatinine $(\mathrm{mmol} / \mathrm{mmol})(\mathrm{e})$, and urea/creatinine $(\mathrm{mmol} / \mathrm{mmol})(\mathrm{f})$. 
TABLE 4: Multiple Regression analysis of total voided volume/4 hrs.

\begin{tabular}{|c|c|c|c|}
\hline Independent variables & $\beta$ & $\mathrm{t}$ & $\mathrm{P}$ value \\
\hline Age (years) & 0.464 & 3.057 & $0.014^{*}$ \\
\hline $\mathrm{BMI}\left(\mathrm{kg} / \mathrm{m}^{2}\right)$ & -0.359 & -1.464 & 0.177 \\
\hline Dietary sodium intake (g/day) & -0.006 & -0.030 & 0.976 \\
\hline Hp concentration $(\mathrm{mmol} / \mathrm{L})$ & 0.540 & 1.931 & 0.086 \\
\hline Urinary potassium excretion $(\mathrm{mmol} / 4 \mathrm{hrs})$ & 0.029 & 0.117 & 0.909 \\
\hline Urinary urea excretion $(\mathrm{mmo} / 4 \mathrm{hrs})$ & -0.688 & -2.716 & $0.024^{*}$ \\
\hline Urinary creatinine excretion ( $\mathrm{mmol} / 4 \mathrm{hrs})$ & 0.872 & 2.321 & $0.045^{*}$ \\
\hline Hp phenotype Hp1-1 [1] vs. rest [2] & 0.673 & 2.744 & $0.023^{*}$ \\
\hline Salt sensitivity phenotype SS [1], SM [2] vs. SR [3] & 0.654 & 3.582 & $0.006^{*}$ \\
\hline
\end{tabular}

Dependent variable: total voided volume $/ 4$ hrs. Data are expressed as standardized regression coefficients $(B), t$-value and $P$ values. $* p<0.05$. NS $=$ not significant. The bold value is the significant $p$ value for age.

osmolality and reduced FWC in healthy young men with a chronic high dietary salt intake ( $12 \mathrm{~g} /$ day) compared with those with a low dietary salt intake (6g/day) [27]. Increased urinary sodium excretion was counterbalanced by a reduction in urea and potassium excretion. Even though no significant increase in urine volume was observed in the high salt intake compared to the low salt intake, urine volume was significantly higher in the highest urinary sodium excreting individuals compared to the lowest, which is in line with the results of our study. Similar results were reported in other studies $[29,30]$. Another study that investigated the effect of varying hydration levels on urinary excretion of sodium after an acute salt load $(5 \mathrm{~g})$ reported an increase in sodium excretion and urinary flow rate in high hydration compared to low hydration status [28]. Excretion of urea increased in high hydration status and decreased in low hydration status, whereas potassium excretion remained unchanged [28]. Under the similar conditions of low hydration status, our study also showed a negative association between the total voided volume and urea excretion. The proposed mechanism behind this is an increase in vasopressin at low hydration level which limits the renal ability to excrete excess sodium selectively and rapidly, thus possibly causing a temporary sodium retention [28].

Beyond dietary intake, diurnal variations in urinary sodium excretion (low in early morning and high in afternoon to night hours) in free living individuals [31] as well as intra-individual variations (e.g., effect of rhythmic secretion of glucocorticoid and mineralocorticoid hormones [27], age, sex, genetic) have been discussed earlier [32]. In the present study, the impact of diurnal variability at the baseline was mainly controlled by having each study participant acting as his own control. The study was conducted under a highly controlled environment to minimize the impact as much as possible by recruiting only young, healthy men after an overnight fast and sober state.

In our previously published study, we demonstrated that Hp polymorphism-related salt sensitivity in Hp1-1 phenotype lacked circadian rhythm in diuresis, renal clearance of free water, and sodium, in contrast to Hp2-2/2-1 phenotypes [18]. $\mathrm{Hp}$ is a haemoglobin clearing plasma protein that displays three major phenotypes in humans as $\mathrm{Hp} \mathrm{1-1,} \mathrm{Hp} 2$ 2, and Hp 2-1 [33], and Hp1-1 has been associated in high BP and salt sensitivity [34]. Results of the current study suggest that subjects with $\mathrm{Hp} 2-2 / \mathrm{Hp} 2-1$ phenotypes could excrete excess sodium efficiently by increasing urine volume after a salt load, possibly due to their salt resistant nature.

Therefore, major genetic and also baseline dietary intake differences between the different study populations may also play an important role in the renal ability to excrete sodium. For an example, a study done by Yatabe et al. reported that urinary sodium excretion reflected the changes in abrupt dietary salt intake at both $3 \mathrm{~g} /$ day and $20 \mathrm{~g}$ /day salt-diet in Japanese subjects ( $m e n=5$, female $=9,21-26$ years) [35], which was incompatible to the present study results. Japanese have a much higher average daily salt intake $9.9 \mathrm{~g}$ [36] than Belgians, who have an average daily salt intake of $5.8 \mathrm{~g}$ [37]. On the other hand, Japanese population has a strongly different $\mathrm{Hp}$ phenotype distribution than the Belgian population. In Japan, $\mathrm{Hp} 1$ allele frequency is about 0.25 (one of the lowest in the world), whereas the Belgian population has a Hp1 allele frequency of 0.40 [33]. This implies that salt sensitive Hp 1-1 subjects are very rare in Japan, whereas in Belgium they represent $\pm 17 \%$ of the population [38]. Therefore, in Japanese population (with a low salt sensitivity), a steep sodium gradient is needed to observe differences ( $3 \mathrm{vs} 20 \mathrm{~g}$ of salt as studied by Yatabe et al.), and this is not the case in Belgian population, which is much more salt-sensitive.

Taken together, the findings of these studies indicate the complex nature of renal sodium handling whilst regulating body water and electrolyte homeostasis in different populations and individuals.

4.2. Relevance and Implementation. Dietary salt restriction is widely recommended as a primary lifestyle intervention in the management of multiple conditions such as high BP, diabetes mellitus, and nocturia $[10,11]$. In nocturnal polyuria (NP), one of the main causes of nocturia [39], an impaired circadian rhythm in renal handling of free water (vasopressin related) or sodium (renin-angiotensin-aldosterone-system (RAAS) and natriuretic peptides related) leads to excessive water and/or sodium diuresis at night $[7,40]$. Dietary salt restriction has been reported to reduce nocturnal urine volume in renal allograft recipients with NP [12]. Thus, dietary salt restriction is a potential beneficial lifestyle modification for NP patients who lost circadian 
rhythm in renal handling of sodium and thus show enhanced sodium diuresis during nighttime [39]. However, the results of our current study suggest that general dietary modification recommendations such as salt reduction might not be beneficial for each patient. People with a low rate of sodium excretion or salt-sensitive individuals produce lower urine volumes as they may retain sodium along with water due to their impaired ability to excrete sodium. In contrast, subjects who are more salt-resistant may efficiently excrete excess sodium with a rapid increase in diuresis. Accordingly, understanding these individual variations in sodium handling is necessary for managing NP, with the emphasis on more personalized dietary recommendations, depending on the patients' salt-sensitivity profile.

Moreover, the European Association of Urology (EAU) recommendations for lifestyle interventions and type and amount of fluid intake in patients with voiding disorders needs further investigation. In this present study, we observed that pure water load induced fast diuresis, whereas water and salt load initially reduced diuresis and promoted late increase in diuresis. Taken together, electrolyte composition of drinks and time of consumption are other important aspects to consider when advising evening fluid intake in NP patients to promote prolonged fluid retention and to maintain fluid balance for an extended period. Likewise, age-related loss of circadian rhythm of renal sodium and water handling is the main cause of NP in the older population [7]. In conclusion, simple dietary modifications, regarding salt and fluid intake, would be beneficial for the overall health in the older population.

4.3. Study Limitations. Body sodium and fluid homeostasis are regulated by a complex, integrated neuro-hormonal system. However, in the present study, we did not measure $\mathrm{BP}$ and the effect on dipping patterns was not studied, which is an important limitation of this study. Furthermore, the results of the present analysis was limited to the acute effect of salt/water load over the following $4 \mathrm{hrs}$. However, with the current study design, it was too challenging to conduct the tests beyond $4 \mathrm{hrs}$, as the study participants were dehydrated and fasted for an extended period of time. In addition, the present study was a pilot study with relatively small sample size and conducted in young healthy men to avoid any kind interference regarding sodium and fluid homeostasis (e.g., chronic illness, hormonal shifts in women that could influence water handling) and in the morning to avoid any practical difficulties in baseline standardization. Thus, results may not be completely generalizable to other populations, particularly the elderly and chronically ill patients with impaired circadian rhythm, renal handling of water or sodium, or affected RAAS.

4.4. Future Research. Nevertheless, the results of this study provide important insights for well-designed future prospective studies. The primary focus of these future projects investigating the renal concentration ability to excrete excess sodium in NP patients should be building more refined individualized lifestyle recommendations. Therefore, future prospective studies with bigger sample size in a real life setting, without predefined fixed-time points for nighttime urine collection, together with frequency volume charts, BP measurements, the effect of dipping patterns, and hormonal analysis, are needed to further understand the role of low-, moderate-, and high-salt evening diets in NP patients in relation to salt sensitivity phenotypes.

\section{Conclusions}

In the present study, we demonstrated that in healthy young men, an acute high-salt load significantly increased diuresis compared to a moderate salt load, with a delay in diuresis effect by $4 \mathrm{hrs}$. Total voided volume was significantly lower in the salt-sensitive phenotype, also described as subjects with low rate of sodium excretion. Consistently, increased voided volume was significantly associated with salt resistance or less-salt-sensitive individuals, age, $\mathrm{Hp} 2-2 / 2-1$ phenotypes, increased urinary creatinine excretion, and decreased urea excretion. Dietary salt restriction is considered as a potential beneficial lifestyle modification for NP patients who lost circadian rhythm in urine production and therefore experience excessive urine production at nighttime. Circadian rhythm of urine production is regulated by the circadian clocks present in the brain, kidney, and bladder. In vivo, an acute high salt load could alter the clock time and drive an unscheduled homeostatic response. The findings of the present study reveal important considerations for future prospective trials which aim to build effective, personalized lifestyle recommendations for NP patients based on saltsensitivity phenotypes.

\section{Data Availability}

The data used to support the findings of this study are available from the corresponding author upon request.

\section{Conflicts of Interest}

K.E reports institutional grants from Ferring and Astellas and grants and other financial contributions from Medtronic outside the submitted work. K.P. receives a research grant from Ferring. The other authors claim no conflicts of interest.

\section{Authors' Contributions}

Conceptualization was done U.S.A. and K.E; methodology was developed by U.S.A., I.V. and K.P.; investigation was performed U.S.A., K.E., J.D., I.V., and K.P.; statistical analysis, data interpretation, original draft preparation were performed by U.S.A; critical review and editing were performed by U.S.A., K.E., J.D., I.V., J.V.C., L.D., K.P., and S.R. All the authors have read and agreed to the final version of the manuscript.

\section{Acknowledgments}

The authors want to thank all the volunteers who participated in this study. Our special thanks to Veerle Decalf and 
Inge Ragolle from the Department of Urology, Ghent University Hospital, for their great assistance in organizing and carrying out the clinical trials. The authors thank the Ghent University Frederik Paulson Chair for unrestricted support.

\section{References}

[1] H. Negoro, A. Kanematsu, K. Yoshimura, and O. Ogawa, "Chronobiology of micturition: putative role of the circadian clock," The Journal of Urology, vol. 190, no. 3, pp. 843-849, 2013.

[2] K. Kessler and O. Pivovarova-Ramich, "Meal timing, aging, and metabolic health," International Journal of Molecular Sciences, vol. 20, no. 8, Article ID 1911, 2019.

[3] J. D. Johnston, J. M. Ordovás, F. A. Scheer, and F. W. Turek, "Circadian rhythms, metabolism, and chrononutrition in rodents and humans," Advances in Nutrition, vol. 7, no. 2, pp. 399-406, 2016.

[4] H. Oike, K. Oishi, and M. Kobori, "Nutrients, clock genes, and chrononutrition," Current Nutrition Reports, vol. 3, no. 3, pp. 204-212, 2014.

[5] U. S. Alwis, J. Delanghe, L. Dossche et al., "Could evening dietary protein intake play a role in nocturnal polyuria?" Journal of Clinical Medicine, vol. 9, no. 8, Article ID 2532, 2020.

[6] H. Hashim, M. H. Blanker, M. J. Drake et al., "International Continence Society (ICS) report on the terminology for nocturia and nocturnal lower urinary tract function," $\mathrm{Neu}$ rourology and Urodynamics, vol. 38, no. 2, pp. 499-508, 2019.

[7] T. F. Monaghan, D. L. Bliwise, M.-A. Denys et al., "Phenotyping nocturnal polyuria: circadian and age-related variations in diuresis rate, free water clearance and sodium clearance," Age and Ageing, vol. 49, no. 3, pp. 439-445, 2020.

[8] C. Gizowski and C. W. Bourque, "Sodium regulates clock time and output via an excitatory GABAergic pathway," Nature, vol. 583, no. 7816, pp. 421-424, 2020.

[9] T. Matsuo, Y. Miyata, and H. Sakai, "Daily salt intake is an independent risk factor for pollakiuria and nocturia," International Journal of Urology, vol. 24, no. 5, pp. 384-389, 2017.

[10] T. Matsuo, Y. Miyata, and H. Sakai, "Effect of salt intake reduction on nocturia in patients with excessive salt intake," Neurourology and Urodynamics, vol. 38, no. 3, pp. 927-933, 2019.

[11] T. F. Monaghan, K. P. Michelson, Z. D. Wu et al., "Sodium restriction improves nocturia in patients at a cardiology clinic," Journal of Clinical Hypertension, vol. 22, no. 4, pp. 633-638, 2020.

[12] Y. Okumura, K. Asai, T. Kobayashi et al., "Dietary sodium restriction reduces nocturnal urine volume and nocturnal polyuria index in renal allograft recipients with nocturnal polyuria," Urology, vol. 106, pp. 60-64, 2017.

[13] M. Kanbay, Y. Chen, Y. Solak, and P. W. Sanders, "Mechanisms and consequences of salt sensitivity and dietary salt intake," Current Opinion in Nephrology and Hypertension, vol. 20, pp. 37-43, 2012.

[14] P. Rust and C. Ekmekcioglu, "Impact of salt intake on the pathogenesis and treatment of hypertension," Advances in Experimental Medicine \& Biology, vol. 2, pp. 61-84, 2017.

[15] D. Majid, M. Prieto, and L. Navar, "Salt-sensitive hypertension: perspectives on intrarenal mechanisms," Current Hypertension Reviews, vol. 11, no. 1, pp. 38-48, 2015.
[16] M. H. Weinberger, "Salt sensitivity as a predictor of hypertension," American Journal of Hypertension, vol. 615S-16S, 1991.

[17] G. Kimura, Y. Dohi, and M. Fukuda, "Salt sensitivity and circadian rhythm of blood pressure: the keys to connect CKD with cardiovasucular events," Hypertension Research, vol. 33, no. 6, pp. 515-520, 2010.

[18] U. S. Alwis, S. Roggeman, T. F. Monaghan, M. A. Denys, K. Everaert, and J. Delanghe, "Haptoglobin 1 - 1 phenotype: a risk factor for 24 - hours polyuria," International Journal of Clinical Practice, vol. 73, Article ID e13419, 2019.

[19] M. A. Elorza, A. Garcia, A. Fuster, and A. Muller, "Evaluation of the "Auto-Stat 6010" automatic osmometer and its comparison with the "Digimatic-Advanced 3DII" manual osmometer," European Journal of Clinical Chemistry and Clinical Biochemistry, vol. 31, pp. 245-249, 1993.

[20] B. Wuyts, D. Bernard, N. Van Den Noortgate et al., "Reevaluation of formulas for predicting creatinine clearance in adults and children, using compensated creatinine methods," Clinical Chemistry, vol. 49, no. 6, pp. 1011-1014, 2003.

[21] E. J. Sampson and M. A. Baird, "Chemical inhibition used in a kinetic urease/glutamate dehydrogenase method for urea in serum," Clinical Chemistry, vol. 25, no. 10, pp. 1721-1729, 1979.

[22] A. Von Eckardstein, H. J. Roth, G. Jones et al., "Cobas 8000 modular analyzer series evaluated under routine-like conditions at 14 sites in Australia, Europe, and the United States," Journal of Laboratory Automation, vol. 18, no. 4, pp. 306-327, 2013.

[23] O. Smithies, "Zone electrophoresis in starch gels: group variations in the serum proteins of normal human adults," Biochemical Journal, vol. 61, no. 4, pp. 629-641, 1955.

[24] P. C. Fink, M. Römer, R. Haeckel et al., "Measurement of proteins with the Behring Nephelometer. A multicentre evaluation," Journal of Clinical Chemistry and Clinical Biochemistry, vol. 27, pp. 261-276, 1989.

[25] F. Dati, G. Schumann, L. Thomas, F. Aguzzi, S. Baudner, and J. Bienvenu, "Consensus of a group of professional societies and diagnostic companies for interim reference ranges for 14 proteins in serum based on the standardization against the IFCC/BCR/CAP Reference Material (CRM470). International Federation of Clinical Chemistry. Community Bureau of Reference of the commission of the European Communities. College of American Pathologists," European Journal of Clinical Chemistry and Clinical Biochemistry, vol. 34, pp. 517-520, 1996.

[26] S. Vandevijvere, W. De Keyzer, J. P. Chapelle et al., "Estimate of total salt intake in two regions of Belgium through analysis of sodium in $24 \mathrm{~h}$ urine samples," European Journal of Clinical Nutrition, vol. 1-6, 2010.

[27] N. Rakova, K. Kitada, K. Lerchl et al., "Increased salt consumption induces body water conservation and decreases fluid intake," Journal of Clinical Investigation, vol. 127, no. 5, pp. 1932-1943, 2017.

[28] G. Choukroun, F. Schmitt, F. Martinez, T. B. Drüeke, and L. Bankir, "Low urine flow reduces the capacity to excrete a sodium load in humans," American Journal of Physiology Regulatory, Integrative and Comparative Physiology, vol. 273, no. 5, pp. R1726-R1733, 1997.

[29] F. J. He, N. D. Markandu, G. A. Sagnella, and G. A. MacGregor, "Effect of salt intake on renal excretion of water in humans," Hypertension, vol. 38, no. 3, pp. 317-320, 2001. 
[30] F. Cuzzola, F. Mallamaci, G. Tripepi, S. Parlongo, S. Cutrupi, and A. Cataliotti, "Urinary adrenomedullin is related to ET-1 and salt intake in patients with mild essential hypertension," American Journal of Hypertension, vol. 14, no. 3, pp. 224-230, 2001.

[31] T. Iwahori, H. Ueshima, S. Torii et al., "Diurnal variation of urinary sodium-to-potassium ratio in free-living Japanese individuals," Hypertension Research, vol. 40, no. 7, pp. 658-664, 2017.

[32] EFSA Panel on Dietetic Products, Nutrition, and Allergies (NDA), "Scientific Opinion on dietary reference values for sodium," EFSA Journal, vol. 17, Article ID 5778, 2019.

[33] M. R. Langlois and J. R. Delanghe, "Biological and clinical significance of haptoglobin polymorphism in humans," Clinical Chemistry, vol. 42, no. 10, pp. 1589-1600, 1996.

[34] M. H. Weinberger, J. Z. Miller, N. S. Fineberg, F. C. Luft, C. E. Grim, and J. C. Christian, "Association of haptoglobin with sodium sensitivity and resistance of blood pressure," Hypertension, vol. 10, no. 4, pp. 443-446, 1987.

[35] M. Yatabe, T. Iwahori, A. Watanabe et al., "Urinary sodiumto-potassium ratio tracks the changes in salt intake during an experimental feeding study using standardized low-salt and high-salt meals among healthy Japanese volunteers," Nutrients, vol. 9, no. 9, Article ID 951, 2017.

[36] S. Nomura, D. Yoneoka, S. Tanaka et al., "Forecasting disability-adjusted life years for chronic diseases: reference and alternative scenarios of salt intake for 2017-2040 in Japan," BMC Public Health, vol. 20, no. 1, Article ID 1475, 2020.

[37] K. De Ridder and E. S. Teppers, Food Consumption Survey 2014-2015, S. Bel and J. Toreau, Eds., Report 4, WIV-ISP, Brussels, Belgium, 2016.

[38] F. C. Luft, J. Z. Miller, C. E. Grim et al., "Salt sensitivity and resistance of blood pressure. Age and race as factors in physiological responses," Hypertension, vol. 17, no. 1_Suppl, Article ID I102, 1991.

[39] M. Oelke, S. De Wachter, M. J. Drake et al., "A practical approach to the management of nocturia," International Journal of Clinical Practice, vol. 71, no. 11, Article ID e13027, 2017.

[40] P. M. Mckeigue and J. M. Reynard, "Relation of nocturnal polyuria of the elderly to essential hypertension," The Lancet, vol. 355, no. 9202, pp. 486-488, 2000. 